\title{
INVESTIGATION OF STRUCTURAL CHANGES IN METALLIC GLASS $\mathrm{Fe}_{18} \mathrm{Co}_{60} \mathrm{Si}_{9} \mathrm{~B}_{13}$
}

\author{
E. JaKubCZYK, J. FilipeCKI, Z. MANDECKi aNd M. JAKUBCZYK \\ Institute of Physics, Pedagogical University \\ Al. Armii Krajowej 13/15, 42-201 Częstochowa, Poland
}

\begin{abstract}
Structural changes accompanying the isothermal annealing of the metallic glass $\mathrm{Fe}_{18} \mathrm{Co}_{60} \mathrm{Si}_{9} \mathrm{~B}_{13}$ were investigated by $\mathrm{X}$-ray diffraction, positron annihilation as well as by electrical resistivity and the Hall resistivity measurements. It has been found out that at the temperature of $673 \mathrm{~K}$ the complete crystallization did not occur, but only a few nuclei of the $\alpha$-Fe phase, which did not grow, were formed.
\end{abstract}

PACS numbers: $61.43 . \mathrm{Dq}$

\section{Introduction}

Structural changes in metallic glasses and their influence on physical properties are still intensively investigated. The properties that are particularly sensitive to the structural changes are: elasticity (Young modulus), volume, coercivity, and electrical resistivity [1]. Knowledge of conditions under which a material changes its properties, and thus cannot perform its function, gives information about the limits of its application. It was shown in many works that the method of production of the metallic glasses leads to different initial, structural, and energy states, and determines their metastability. Annealing of metallic glasses below the crystallization temperature modifies the short range ordering. Irreversibility is characteristic of majority of those changes. The changes are connected with the topological short range ordering (TSRO) and they reflect the gradual decrease in free volumes specific for metallic glasses. On the other hand, the reversible changes resulting from the periodic change of the sample temperature are connected with the structural changes known as the chemical short range ordering (CSRO) [1-3]. According to the CSRO model the local changes occur in the region nearest to the atom. In many cases both changes take place simultaneously. They can be distinguished by determination of activation energies corresponding to them [3].

Annealing of the amorphous material above the crystallization temperature results in distinct changes of the structure, which change ordering from the short range to the long range. 


\section{Experiment}

The investigated metallic glass $\mathrm{Fe}_{18} \mathrm{Co}_{60} \mathrm{Si}_{9} \mathrm{~B}_{13}$ was produced by the roller quenching method in the Institute of Materials Engineering of Warsaw Technical University. The Hall resistivity and electrical resistivity were measured and the atomic structure was determined by the X-ray diffraction for as-received and annealed samples. The samples were isothermally annealed in the argon atmosphere at the temperature of $673 \mathrm{~K}$ for $10^{2}, 10^{3}, 10^{4}$ and $2 \times 10^{4} \mathrm{~s}$. Each as-received sample underwent the process of annealing. In this paper, lifetime of the positrons was measured as well. Annealing of the samples to measure the lifetime of positrons was carried out at the temperatures of $573 \mathrm{~K}$ and $773 \mathrm{~K}$ for different time intervals $\left(1-10^{5} \mathrm{~s}\right)$.

The Hall voltage was measured by constant current method in constant magnetic field. The samples used in those measurements had five electrodes. Two of them were used for supplying the samples with a constant current along its length. The other three were used for the Hall voltage measurement and ensured elimination of electrode asymmetry. The samples were prepared by the selective etching using photolitography. For the same samples electric resistivity was also measured at a constant current.

The X-ray measurements were performed by DRON-2.0 diffractometer equipped with a horizontal goniometer GUR-5. The applied X-ray tube had a molybdenium target $\left(\lambda_{K_{\alpha}}=0.71069 \times 10^{-10} \mathrm{~m}\right)$ and a graphite monochromator in the primary beam.

Measurements of positron lifetimes were carried out with an ORTEC spectrometer of resolution FWHM $=270$ ps. A ${ }^{22} \mathrm{Na}$ isotope of $3.7 \times 10^{9} \mathrm{~Bq}$ activity was used as a positron source. Each specimen consisted of a few ribbons $15 \mathrm{~mm}$ wide and $0.04 \mathrm{~mm}$ thick up to the total size of $15 \mathrm{~mm} \times 15 \mathrm{~mm} \times 0.25 \mathrm{~mm}$. The source was placed between two identical investigated samples, forming a "sandwich" system.

\section{Results and discussion}

Investigation of the samples of metallic glass $\mathrm{Fe}_{18} \mathrm{Co}_{60} \mathrm{Si}_{9} \mathrm{~B}_{13}$ annealed at different temperatures $(573,673,723,773$ and $823 \mathrm{~K})$ during the constant time period of $4 \mathrm{~h}$ showed that after annealing at $673 \mathrm{~K}$ a small amount of the $\alpha$-Fe phase was formed within the amorphous phase (Fig. 1). Distinct increase in this phase is caused by annealing at the temperature $723 \mathrm{~K}$. Taking into account atomic composition of the sample, we may draw a conclusion that it is a solid solution $\mathrm{FeCoSi}$ on the basis of the $\alpha$-Fe phase. After annealing of the samples at the temperature of $773 \mathrm{~K}$ the $\mathrm{Co}_{2} \mathrm{~B}$ phase with the tetragonal structure is crystallized from the rest of the amorphous phase. It is also possible that the $\mathrm{Fe}_{2} \mathrm{~B}$ phase with the same tetragonal structure is crystallized. The peaks of the $\mathrm{Co}_{2} \mathrm{~B}$ and $\mathrm{Fe}_{2} \mathrm{~B}$ phases overlap and therefore unambiguous interpretation of these peaks is difficult [4].

In Fig. 1 the X-ray diffraction pattern of the sample annealed at the temperature of $573 \mathrm{~K}$ is not given because it has a diffusive "halo" characteristic of the amorphous phase. 


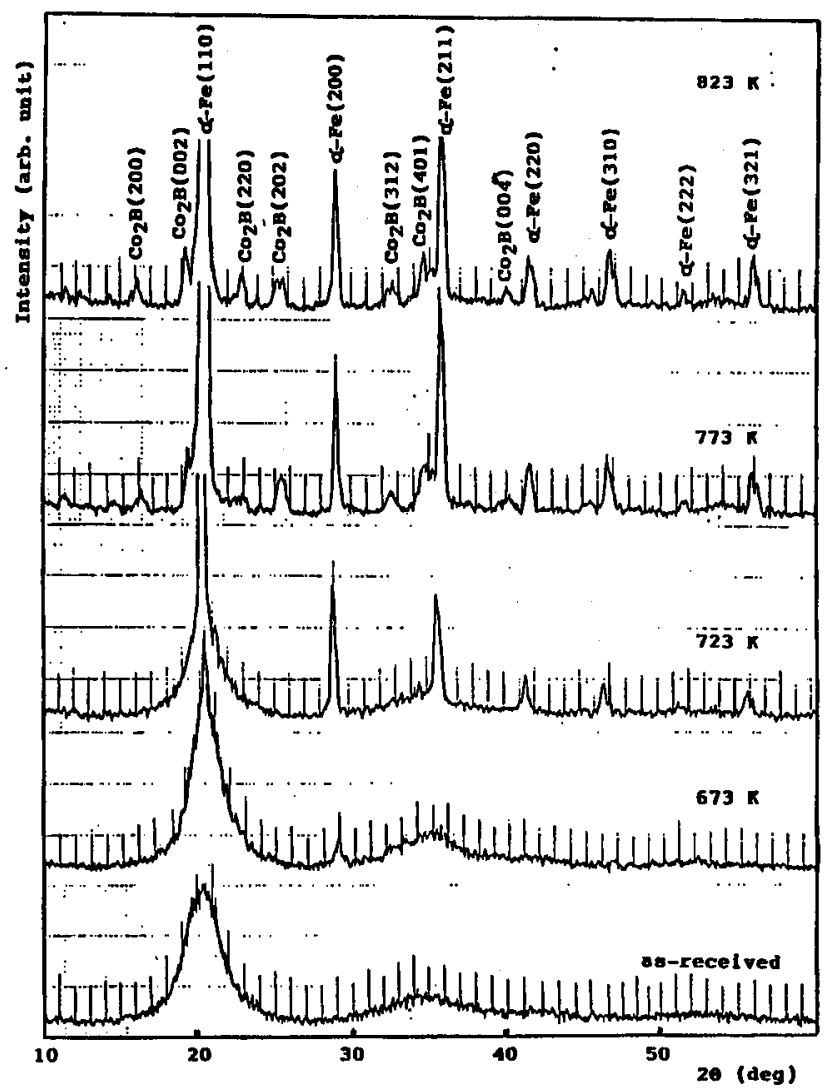

Fig. 1. X-ray diffraction patterns for samples of the $\mathrm{Fe}_{18} \mathrm{Co}_{60} \mathrm{Si}_{9} \mathrm{~B}_{13}$ alloy annealed at different temperatures during $4 \mathrm{~h}$.

Also, measurements of the electrical resistivity and the Hall voltage for the samples annealed at temperature of $573 \mathrm{~K}$ did not show any changes in comparison with the values of those parameters for the as-received sample. However, those values change distinctly when crystallization occurs in the sample $[1,5]$.

To observe changes in the metallic glass $\mathrm{Fe}_{18} \mathrm{Co}_{60} \mathrm{Si}_{9} \mathrm{~B}_{13}$ before the crystallization the $\mathrm{X}$-ray diffraction patterns, electrical resistance and the Hall voltage were measured for the samples annealed at the temperature of $673 \mathrm{~K}$ during different time intervals ranging from $10^{2}$ to $2 \times 10^{4} \mathrm{~s}$. The X-ray diffraction patterns presented in Fig. 2 prove that annealing of the sample for $10^{4} \mathrm{~s}$ begins creation of the $\alpha$-Fe phase. Moreover, annealing for $10^{3} \mathrm{~s}$ leads to the changes in diffusive "halo" which is a proof of atomic rearrangements which give a small number of crystallites after annealing for $10^{4} \mathrm{~s}$. Increase in the annealing time increases slightly the amount of those crystallites.

The changes of electrical resistivity are presented in Fig. 3 as relative changes with respect to the resistivity of as-received sample $\rho_{0}\left(\rho_{0}=1.75 \pm 0.02 \mu \Omega \mathrm{m}\right)$. The electrical resistivity decreases with increasing annealing time. It saturates 


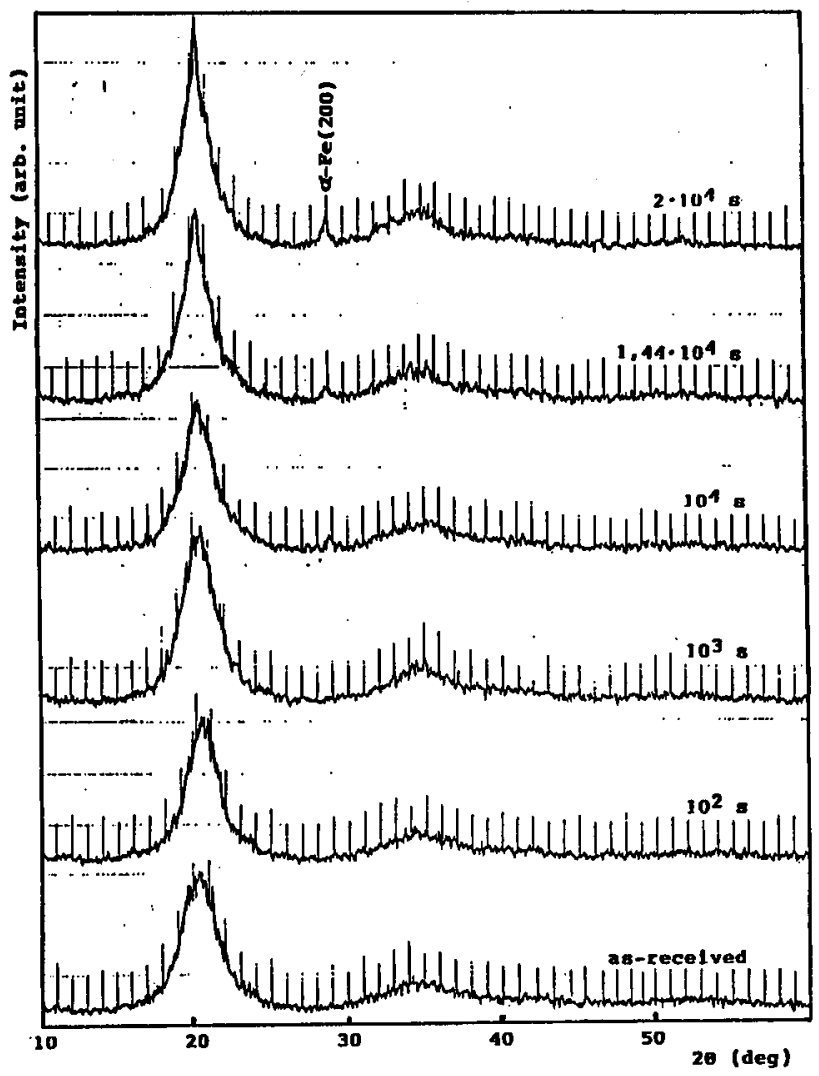

Fig. 2. X-ray diffraction patterns for samples of the $\mathrm{Fe}_{18} \mathrm{Co}_{60} \mathrm{Si}_{9} \mathrm{~B}_{13}$ alloy for different time intervals at a constant temperature of $673 \mathrm{~K}$.

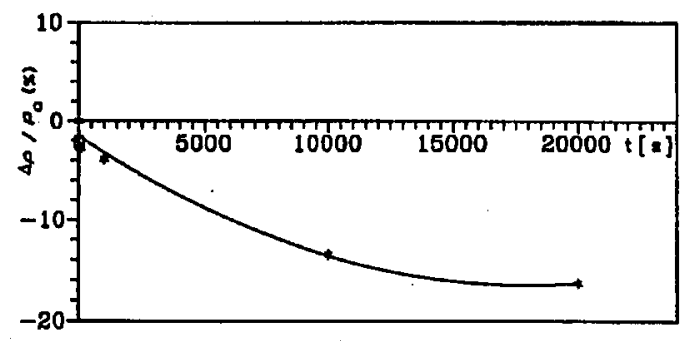

Fig. 3. The relative electrical resistivity $\Delta \rho / \rho_{0}$ as function of annealing time at the temperature of $673 \mathrm{~K}$.

after annealing for $10^{4} \mathrm{~s}$, i.e. when the peak attributed to the formation of the crystalline $\alpha$-Fe phase appears on the X-ray diffractogram (Fig. 2).

In Fig. 4 the Hall resistance $\rho_{\mathrm{H}}$ is shown as a function of the external magnetic induction $B_{0}$ for samples annealed during different time intervals. This dependence is typical of ferromagnetic substances [6]. The Hall resistivity and the electrical 


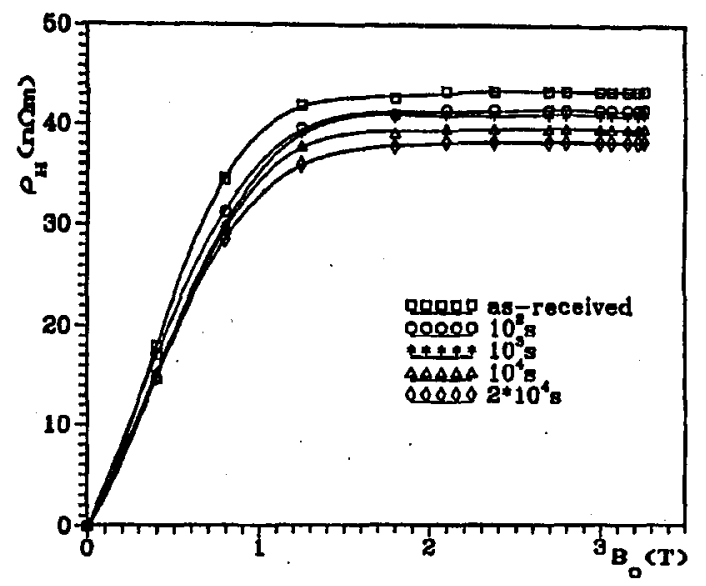

Fig. 4. The Hall resistivity $\rho_{\mathrm{H}}$ as function of the applied magnetic induction $B_{0}$ for $\mathrm{Fe}_{18} \mathrm{Co}_{60} \mathrm{Si}_{9} \mathrm{~B}_{13}$ alloy annealed at temperature of $673 \mathrm{~K}$ during different time intervals.

resistivity decreases as the time of annealing increases. These changes are slight in comparison to the changes caused by formation of the crystalline phase from the amorphous one [5].

The measurements of the lifetime of positrons were carried out for the samples annealed at the temperatures of 573 and $773 \mathrm{~K}$ during different time intervals $\left(1-10^{5} \mathrm{~s}\right)$. The obtained values of the positrons lifetime in amorphous samples correspond to the annihilation of positrons trapped at the monovacancy-sized free volumes. However, the values of the positrons lifetime for crystallized samples indicate occurrence of microvoids (agglomerates of several vacancies) [7-9]. In Fig. 5 the mean values of positron lifetimes are shown. They were calculated using POSITRONFIT computer program, which takes into account the positron source correction and the resolution function [10].

In Fig. $5 \mathrm{a}$ the maximum of the positron lifetime is observed at $10^{3} \mathrm{~s}$ of annealing time. It seems that the positron lifetime in positron trapping regions initially increases and later, after annealing above $10^{3} \mathrm{~s}$, decreases. This fact suggests that this phenomenon is caused by the atomic rearrangements occurring at $573 \mathrm{~K}$ and it is connected with the changes of topological ordering of the amorphous structures (TSRO). Thus, it can be stated that in the investigated metallic glass after annealing at $573 \mathrm{~K}$ the increase in the positron trapping in microdefects related to escalation of the monovacancy-sized free volumes in the amorphous structures can be observed. The calculated values of positron lifetimes after the process of crystallization vs. time are plotted in Fig. 5b. One can observe a distinct decrease in $\tau$. From this fact it is possible to conclude that the decrease in positron lifetimes in positron trapping areas after crystallization is connected with the formation of microvoids in the investigated specimens.

Recapitulating the obtained results one may conclude that annealing at the temperature of $673 \mathrm{~K}$ for $10^{4} \mathrm{~s}$ lies on a borderline between the arrangement of atoms within short and long range ordering. 

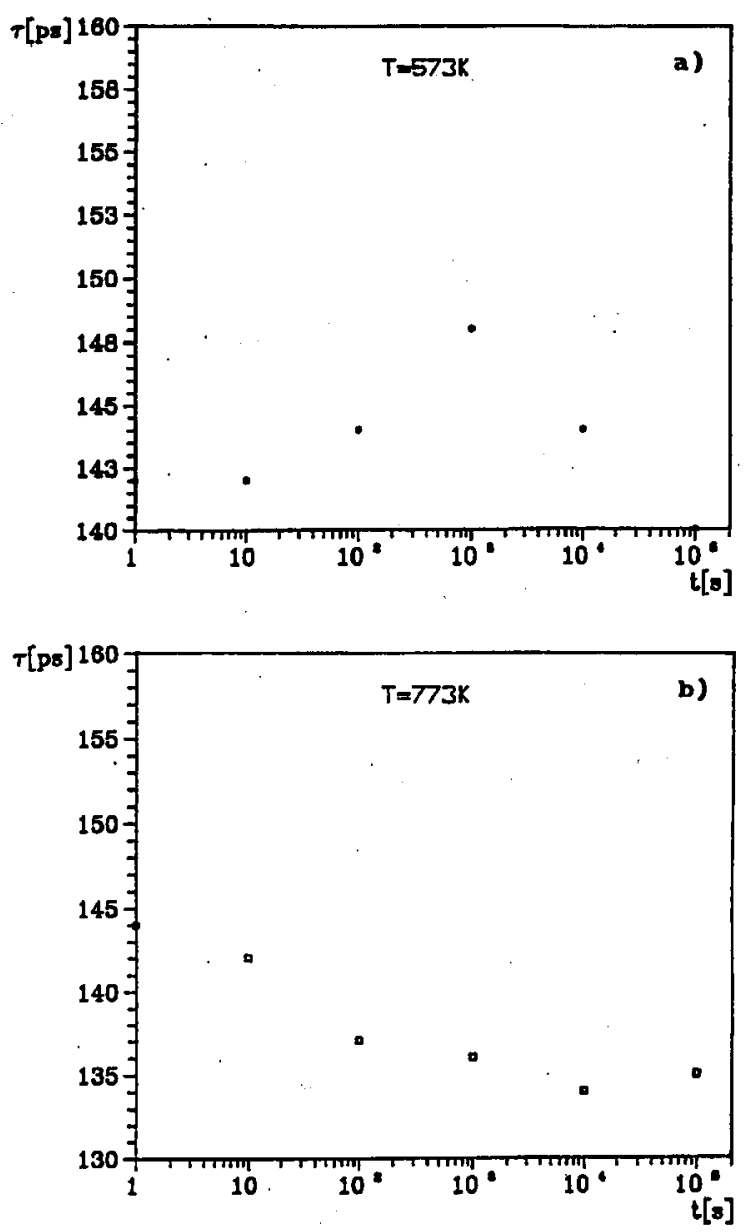

Fig. 5. The values of average positron lifetimes $\tau$ in $\mathrm{Fe}_{18} \mathrm{Co}_{60} \mathrm{Si}_{9} \mathrm{~B}_{13}$ alloy annealed during different time intervals at temperature of $573 \mathrm{~K}(\mathrm{a})$ and $773 \mathrm{~K}(\mathrm{~b})$.

Crystallization of the phase with the $\alpha$-Fe structure requires the smallest activation energy in comparison to the phases $\mathrm{Co}_{2} \mathrm{~B}$ and $\mathrm{Fe}_{2} \mathrm{~B}$. The energy provided by annealing at temperature of $673 \mathrm{~K}$ during $2 \times 10^{4} \mathrm{~s}$ is too small to crystallize completely the $\alpha-\mathrm{Fe}$ phase from the amorphous phase of the investigated metallic glass.

\section{References}

[1] T. Komatsu, Res. Mech. 31, 263 (1990).

[2] G. Riontino, M. Baricco, Philos. Mag. B 61, 715 (1990).

[3] A. Van den Beukel, Key Eng. Mater. 81-83, 3 (1993).

[4] B.D. Cullity, Elements of X-ray Diffraction, Addison-Wesley, London 1959. 
[5] E. Jakubczyk, Z. Mandecki, J. Filipecki, Key Eng. Mater. 81-83, 383 (1993).

[6] K. Heineman, K. Barner, J. Magn. Magn. Mater. 42, 291 (1984).

[7] S. Chen, S.Y. Chuang, Appl. Phys. Lett. 31, 255 (1977).

[8] A. Liolies, M. Dedoussis, M. Chardalas, S. Charalambous, Cryst. Res. Technol. 22, 199 (1987).

[9] R.N. West, Adv. Phys. 22, 23 (1973).

[10] P. Kirkegaard, M. Eldrup, Comp. Phys. Commun. 23, 307 (1981). 